\title{
Interplay between topological and thermodynamic stability in a metastable magnetic skyrmion lattice
}

\author{
Hiroshi Oike ${ }^{1}$, Akiko Kikkawa', Naoya Kanazawa ${ }^{2}$, Yasujiro Taguchi ${ }^{1}$, Masashi Kawasaki ${ }^{1}$, , \\ Yoshinori Tokura ${ }^{1,2}$ and Fumitaka Kagawa ${ }^{1,3 \star}$
}

Topologically stable matter can have a long lifetime, even if thermodynamically costly, when the thermal agitation is sufficiently low ${ }^{1,2}$. A magnetic skyrmion lattice (SkL) represents a unique form of long-range magnetic order that is topologically stable $^{3-9}$, such that a long-lived, metastable SkL can form. Experimental observations of the SkL in bulk crystals, however, have mostly been limited to a finite and narrow temperature region in which the SkL is thermodynamically stable $e^{5,7,10-14}$; thus, the benefits of the topological stability remain unclear. Here, we report a metastable SkL created by quenching a thermodynamically stable SkL. Hall-resistivity measurements of MnSi reveal that, although the metastable SkL is short-lived at high temperatures, the lifetime becomes prolonged ( $\gg 1$ week) at low temperatures. The manipulation of a delicate balance between thermal agitation and the topological stability enables a deterministic creation/annihilation of the metastable SkL by exploiting electric heating and subsequent rapid cooling, thus establishing a facile method to control the formation of a SkL.

The perspective of topology provides useful insights into the lifetime of a many-body system that is not in the most thermodynamically stable form. In an aggregate of carbon atoms, for instance, whereas the most thermodynamically stable form at standard temperatures and pressures is graphite, other forms, such as diamond and fullerene-molecule crystals, also have long lifetimes, despite a thermodynamic free-energy penalty ${ }^{15}$. From a topological perspective $^{16}$, the carbon networks of the crystalline allotropes are topologically distinct from each other. Therefore, diamond and fullerene-molecule crystals are unable to transform into graphite unless thermal agitation enables the breaking and reformation of the carbon networks. States with this absence of continuity in topology are often termed topologically protected, or topologically stable, and this condition guarantees a finite lifetime for the thermodynamically less stable states of matter, thereby qualitatively accounting for their metastable nature.

Despite the many examples of topologically distinct crystals $^{16}$, only recently has a topologically stable long-range magnetic order been identified experimentally in the form of a magnetic SkL, which is a hexagonal arrangement of magnetic vortex lines ${ }^{5-9}$ (for the schematic spin configuration, see Fig. 1a). Because of its topology, the SkL is topologically protected from other competing nontopological spin configurations, such as ferromagnetic and spinconical orders (Fig. 1a), thus providing a unique platform to observe how long a topologically stable long-range ordering of spins can persist beyond its thermodynamic stability. To address this issue, the lifetime of a metastable SkL requires investigation. In reality, however, unless randomly positioned dopant atoms ${ }^{6}$ or pressure inhomogeneities ${ }^{17}$ are introduced, the SkL in various chiral magnets is readily annihilated when the SkL becomes thermodynamically costly by passing through the SkL-to-conical transition line $e^{5,7,10-14}$ (for a typical phase diagram, see Fig. 2a). Thus, the intrinsic lifetime of the metastable SkL remains unknown.

In pursuing a metastable SkL that should potentially form even in a disorder-free system, we noted that, in the creation process of natural diamonds, thermodynamically stable diamonds grown in the mantle are quenched when they move to the surface of the Earth and, hence, the diamond-to-graphite transition is kinetically avoided. On the basis of this realization, we envisaged that the SkL-to-conical transition, which normally occurs during cooling, can also be kinetically avoided if the cooling rate is sufficiently high (Fig. 1a). To test this working hypothesis, we chose nominally dopant-free $\mathrm{MnSi}$ as an archetypal system of a skyrmion-hosting material and exploited rapid cooling following electric-pulse heating applied to the sample. For instance, when a rectangular current pulse of $3.1 \times 10^{6} \mathrm{~A} \mathrm{~m}^{-2}$ with a duration of $100 \mathrm{~ms}$ is applied to the sample at $10 \mathrm{~K}$, the sample temperature is observed to quickly increase and reach a quasi-steady state with a temperature of approximately $33-34 \mathrm{~K}$. After the pulse ceases, the sample is rapidly cooled to the environmental temperature at a cooling rate of approximately $700 \mathrm{~K} \mathrm{~s}^{-1}$ (for the estimation of the time-varying sample temperature and the cooling rate, see Supplementary Information and Supplementary Fig. 1).

Using the quenching technique described, the impact of quenching on the magnetic structure can be explored beyond the normally used cooling rate, $10^{-2}-10^{0} \mathrm{~K} \mathrm{~s}^{-1}$. To this end, we focused on the Hall resistivity, $\rho_{y x}$, as a sensitive probe for SkL formation, particularly in $\mathrm{MnSi}$ (refs 18,19), and found that $-\rho_{y x}$ at $10 \mathrm{~K}$ under a magnetic field of $0.22 \mathrm{~T}$ indeed changes from -2 to $32 \mathrm{n} \Omega \mathrm{cm}$ after applying the current pulse described. Whereas the enhanced $\rho_{y x}$ value remains constant as a function of time (verified up to one week), it exhibits transition-like behaviour in response to a slow magnetic field sweep $\left(5.0 \times 10^{-4} \mathrm{~T} \mathrm{~s}^{-1}\right)$ and then decreases to the values corresponding to the equilibrium state (Fig. 1b). These observations are reasonably understood by considering that a longlived metastable magnetic structure is formed as a result of heating and subsequent rapid cooling.

To elucidate the origin of the metastable state, we first examined whether it is actually a quenched state. We tailored the cooling rate with ramp pulses (for details, see Supplementary Information and Supplementary Fig. 1) and observed how the $\rho_{y x}$ value changes. Figure 1c summarizes the $\rho_{y x}$ values measured after applying various cooling rates; remarkably, sharp crossover behaviour is observed from the slowly cooled to the rapidly cooled regimes, thus verifying that the enhanced $\rho_{y x}$ value of approximately $32 \mathrm{n} \Omega \mathrm{cm}$ is indeed a consequence of the quenching. Because neither the ordinary Hall 
a

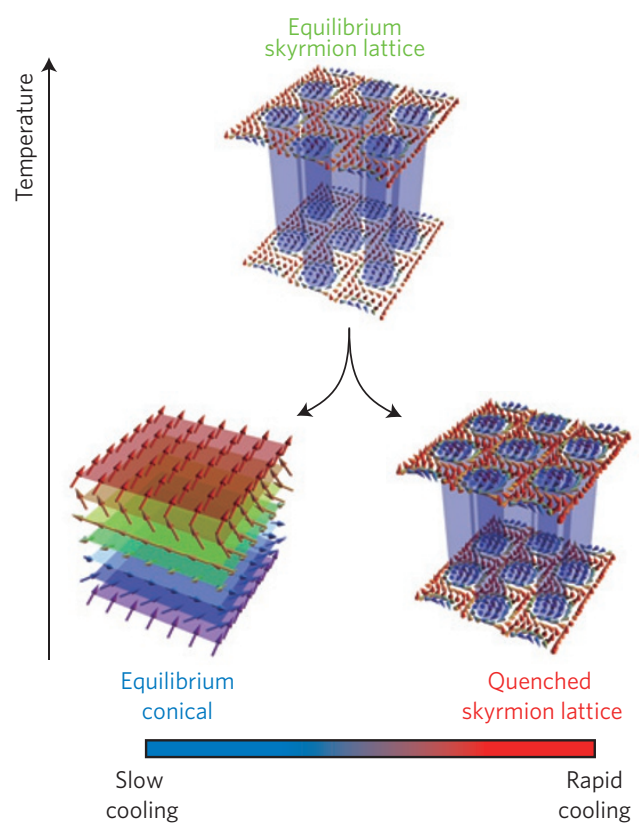

b

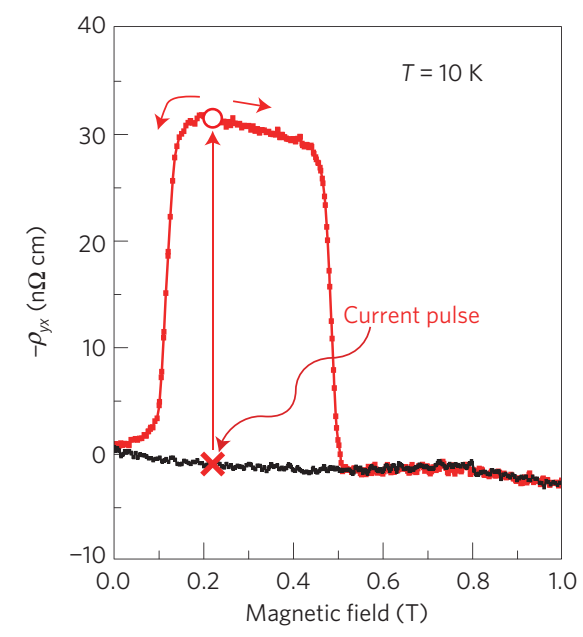

C

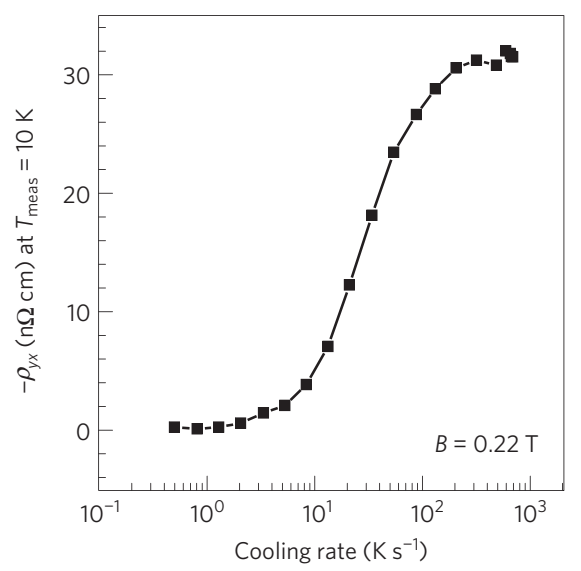

d

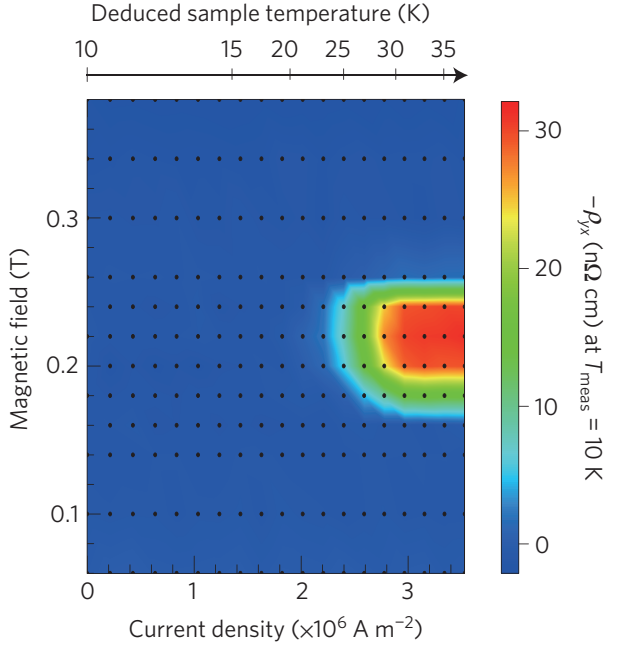

Figure 1 | Metastable skyrmion lattice stabilized under rapid cooling. a, Schematic of the cooling-rate-dependent bifurcation of an equilibrium skyrmion lattice (SkL). Whereas the equilibrium SkL changes into the thermodynamically stable conical phase under slow cooling, rapid cooling causes kinetic avoidance of the SkL-to-conical transition, resulting in a quenched metastable SkL. Under an intermediate cooling rate, a mixture of the two phases is expected. $\mathbf{b}$, Magnetic field dependence of the Hall resistivity, $\rho_{y x}$, measured before and after quenching at $0.22 \mathrm{~T}$. By applying a current pulse to the equilibrium state under a magnetic field of $0.22 \mathrm{~T}$ (the cross), the $\rho_{y x}$ value changes to an enhanced value (the open circle). c, Cooling-rate dependence of $\rho_{y x}$ measured at $10 \mathrm{~K}$ and $0.22 \mathrm{~T}$ after quenching is complete. $\mathbf{d}$, Contour plot of $\rho_{y x}$ measured at $10 \mathrm{~K}$ post-quenching using various current densities under selected magnetic fields. The deduced sample temperature during the electric current application is also indicated on the upper horizontal axis (see also Supplementary Fig. 1). Contour plots measured at 5 and $23 \mathrm{~K}$ are shown in Supplementary Fig. 2.

nor the anomalous Hall resistivities can explain such a greatly enhanced value of $\rho_{y x}$ (Fig. 1b), it is important to consider the contributions of the topological Hall resistivity, which is known to arise in the presence of the SkL because of its real-space Berry phase associated with the non-zero topological winding number ${ }^{18-21}$. In fact, the magnitude of the enhancement, $\Delta \rho_{y x} \approx 31-34 \mathrm{n} \Omega \mathrm{cm}$, is similar to the reported value, $35-37 \mathrm{n} \Omega \mathrm{cm}$, observed when the metastable SkL persists at $10 \mathrm{~K}$ in $\mathrm{MnSi}$ under the influence of pressure inhomogeneity ${ }^{17}$. Given that the low-temperature value of the topological Hall resistivity depends only weakly on pressure and sample quality as long as the metastable SkL is realized ${ }^{17}$, the good agreement between the present and reported values indicates that the SkL is the most likely candidate for the present quenched state.

This scenario is further supported by the observation of an enhanced $\rho_{y x}$ value under various magnetic field/current density conditions. The contour plot of $\rho_{y x}$ measured after the application of a pulse with a given current density is shown in Fig. 1d; here, we find that the enhancement of $\rho_{y x}$ is limited to a magnetic field range of $0.17-0.26 \mathrm{~T}$, which is in good agreement with that of the equilibrium SkL phase in our sample, 0.14-0.27 T (Fig. 2a). Furthermore, the enhancement of $\rho_{y x}$ becomes appreciable only if the deduced sample temperature during the pulse application (see the upper horizontal axis of Fig. 1d) reaches or exceeds the temperatures of the equilibrium SkL (27.0-28.8 K). These findings lead us to conclude that the quenched magnetic state is a metastable SkL caused by the kinetic avoidance of the SkL-to-conical transition. Unexpectedly to us, the metastable SkL is formed even when the sample is quenched from the paramagnetic regime $(>28.8 \mathrm{~K})$, indicating that the paramagnetic-to-SkL transition has faster kinetics than the SkLto-conical transition and, therefore, cannot kinetically be avoided, at least at the present cooling rate, $700 \mathrm{~K} \mathrm{~s}^{-1}$. 
a

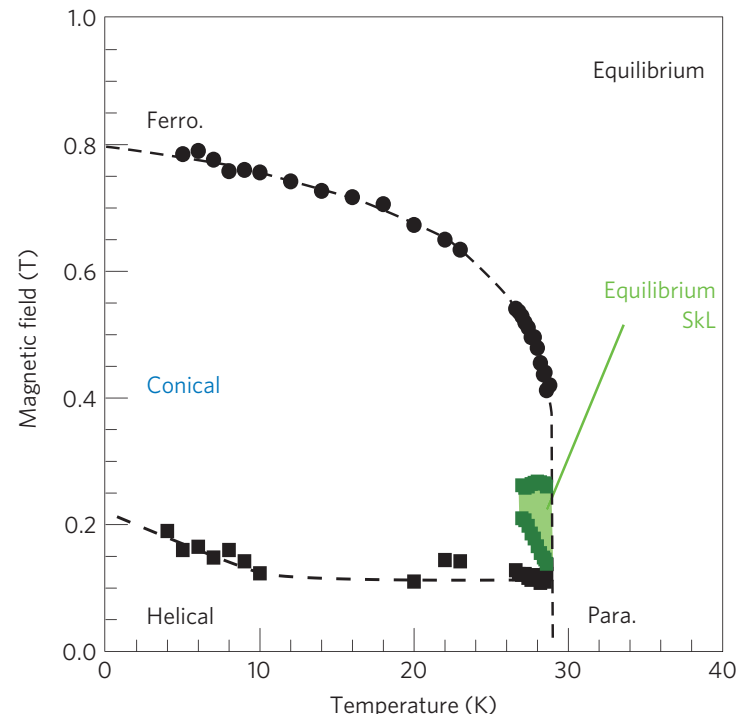

b

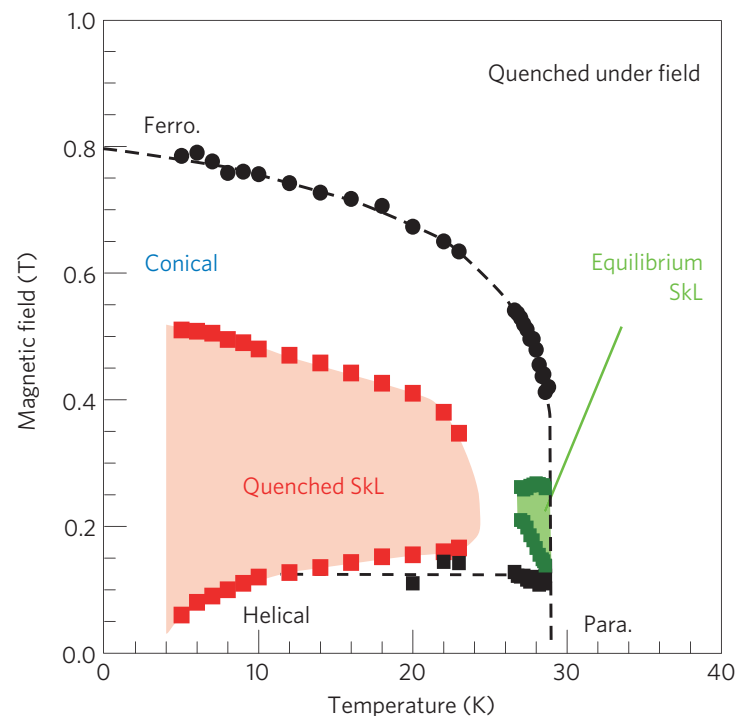

Figure $\mathbf{2}$ | Magnetic state diagrams of $\mathrm{MnSi}$ under equilibrium and quenched conditions. a, Phase diagram under thermodynamic equilibrium. b. Magnetic state diagram including the metastable SkL created by quenching under a magnetic field. Red symbols in $\mathbf{b}$ were determined from the data shown in Fig. 1b and Supplementary Fig. 2. Whereas the boundaries of the equilibrium SkL were determined from the $\rho_{x x}$ data, the ferromagnetic transition line was determined from the $\rho_{y x}$ data (see Supplementary Figs 3 and 4).

Having established a method to create a metastable SkL in a nominally clean sample, we can now address the issue of the stability beyond the equilibrium phase diagram. We found that the long-lived metastable SkL can be created at any temperature between 5 and $23 \mathrm{~K}$ using the same quenching method (see Supplementary Figs 2 and 3); accordingly, we could further examine the stability of the metastable SkL against a magnetic field sweep at each temperature and construct a magnetic state diagram that includes the quenched SkL, as shown in Fig. 2b. Here, two important aspects can be highlighted. First, the temperature/magnetic-field region in which the quenched SkL can persist is significantly extended compared to that of the equilibrium SkL phase, which exemplifies the remarkable stability of the metastable SkL. Second, there is a temperature gap $(\sim 24-27 \mathrm{~K})$ in which neither the equilibrium SkL nor the metastable SkL can persist, implying that the metastable SkL is short-lived in this temperature range. a

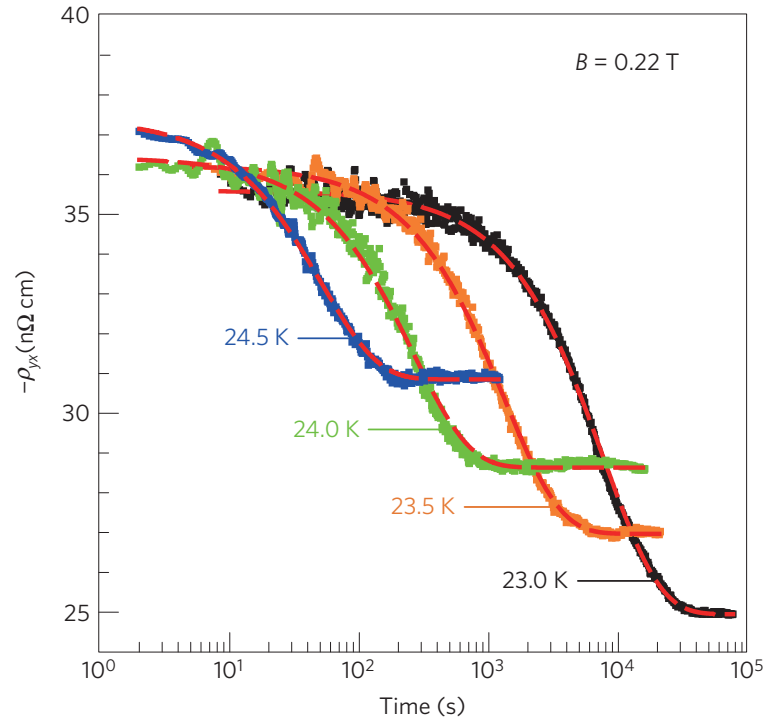

b

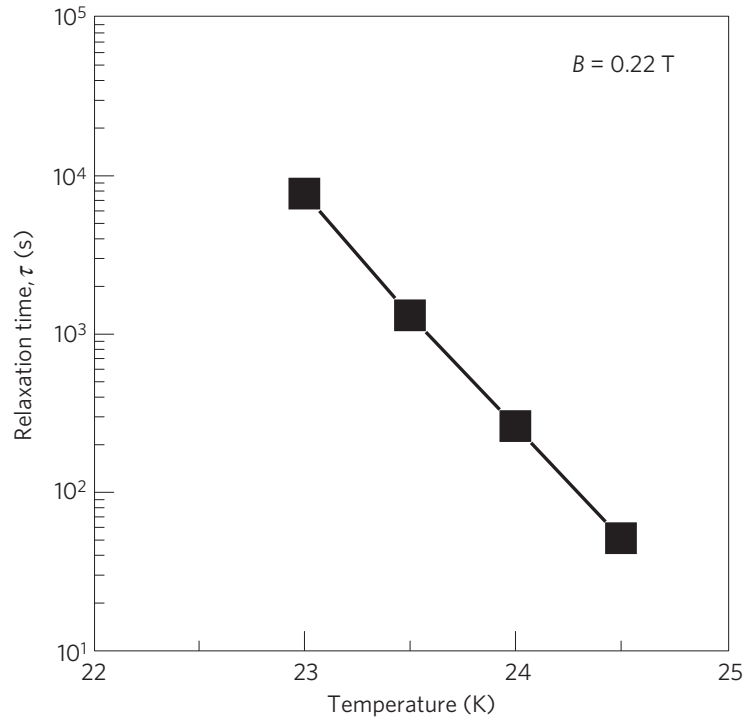

Figure 3 | Lifetime of the metastable skyrmion lattice. a, Time evolution of $\rho_{y x}$ at selected temperatures after quenching at $0.22 \mathrm{~T}$. The broken red lines are fits to the standard relaxation equation (1). b. Temperature dependence of the lifetime of the metastable SkL at 0.22 T. The lifetimes are obtained from the fitting results in $\mathbf{a}$.

Such a short-lived metastable SkL can be substantiated by the time decay of the enhanced $\rho_{y x}$ value, as shown in Fig. 3a. The relaxation of $\rho_{y x}$ to the equilibrium value can clearly be seen, in accord with the expectation that the metastable SkL is short-lived in this temperature range. The behaviour is characterized well by the standard relaxation equation (denoted by the broken lines in Fig. 3a):

$$
\rho_{y x}(t)=\rho_{y x, 0}+\left(\rho_{y x, \infty}-\rho_{y x, 0}\right)\{1-\exp (-t / \tau)\}
$$

where $\rho_{y x, 0}$ and $\rho_{y x, \infty}$ denote the initial and fully relaxed (or equilibrium) values of $\rho_{y x}$, respectively, and $\tau$ represents the relaxation time (or, equivalently, the lifetime of the metastable SkL). Figure $3 \mathrm{~b}$ summarizes the $\tau$ values derived for various temperatures. Note that as the temperature approaches the SkL transition temperature of $27 \mathrm{~K}$, the relaxation time decreases markedly and will probably decrease to less than $10 \mathrm{~s}$. This behaviour reasonably accounts for why the SkL is readily annihilated during cooling in nominally clean systems, even though it is topologically 


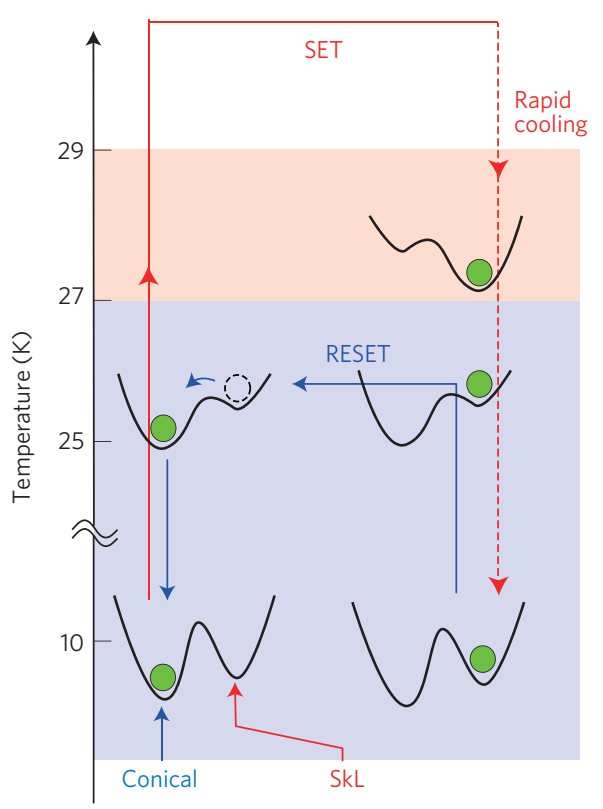

b

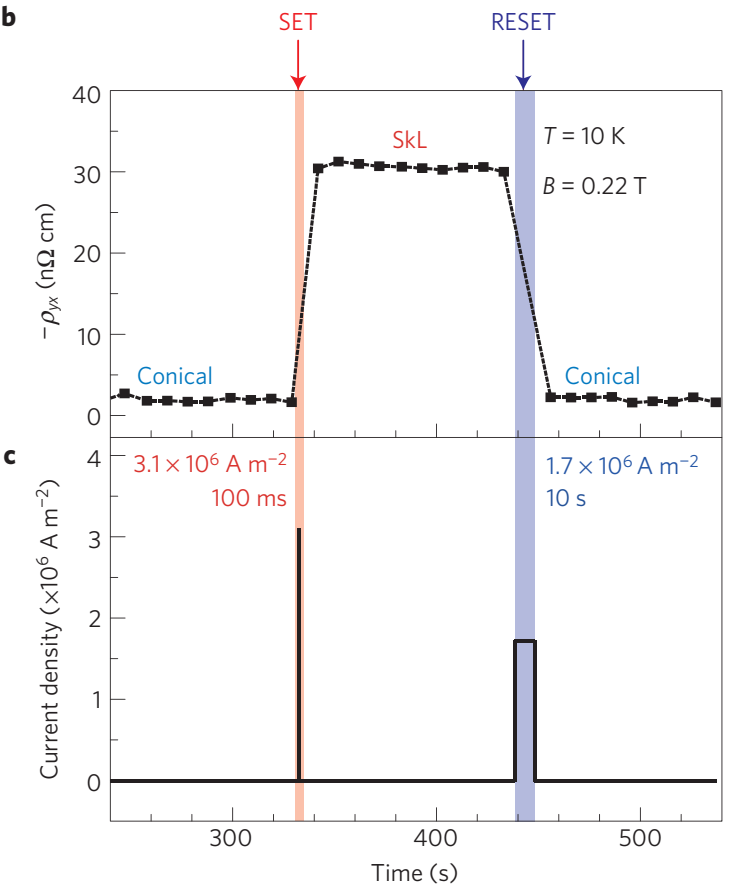

d

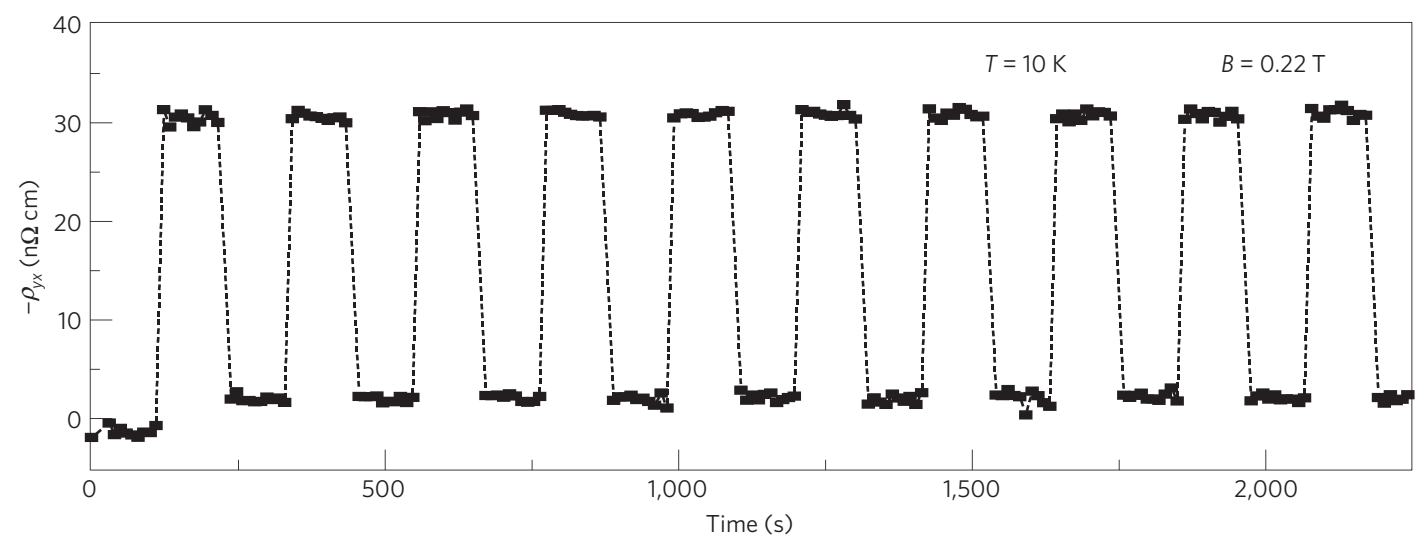

Figure 4 | Creation and annihilation of the metastable skyrmion lattice through thermal control. $\mathbf{a}$, Scheme for repeatable switching between the SkL and the conical phase in terms of a schematic free-energy landscape in a multidimensional spin-configuration space. Note that, whereas the SkL is thermodynamically stable in the red-hatched temperature region $(27-29 \mathrm{~K})$, the conical state is thermodynamically stable in the blue-hatched temperature region $(<27 \mathrm{~K})$. b,c, Single-cycle operation of the switching under the application of rectangular current pulses. The time profiles of $\rho_{y x}$ and the current density are shown in $\mathbf{b}$ and $\mathbf{c}$, respectively. $\mathbf{d}$, Repetitive switching between the metastable SkL and equilibrium conical phases.

stable $e^{5,7,10-14}$. Nevertheless, after quickly passing through the hightemperature region in which the metastable SkL is short-lived, the lifetime becomes unmeasurably long towards low temperatures. Consequently, the quenched SkL is stabilized practically. The significant increase in $\tau$ invokes the thermal activation process and suggests that the SkL-to-conical topology change proceeds via an intermediate spin configuration with a creation energy, $E_{\mathrm{g}}$. In estimating the order of magnitude of $E_{\mathrm{g}}$, we tentatively assume that $E_{\mathrm{g}}$ is dependent on temperature and proportional to the square of the local magnetic moment, $m$. Then, if we adopt a simplified $m$-temperature profile, $m \sim\left(T_{c}-T\right)^{0.5}$, with $T_{c} \approx 29 \mathrm{~K}$ (the SkLparamagnetic transition temperature), $E_{\mathrm{g}}$ at zero temperature is estimated to be $\sim 2 \times 10^{3} \mathrm{~K}$ (for details, see Supplementary Fig. 5). Although the intermediate spin configurations cannot be clearly described from the $\rho_{y x}$ data, the 'emergent magnetic monopoles' discussed in the literature ${ }^{22}$ may be relevant.

Finally, on the basis of the present findings, we demonstrate reversible and deterministic switching between the topologically distinct spin configurations. Figure $4 \mathrm{a}$ shows phenomenological free-energy landscapes of the relevant spin configurations, illustrating the working principles of the switching. In the 'SET process', as has been discussed in Fig. $1 \mathrm{~b}-\mathrm{d}$, the application of a current pulse to the conical state results in the metastable SkL through the kinetic avoidance of the SkL-to-conical transition. In the 'RESET' process, a relatively long current pulse (10 s) with a moderate intensity $\left(1.7 \times 10^{6} \mathrm{~A} \mathrm{~m}^{-2}\right)$ was applied to the quenched SkL to heat the sample to $25-27 \mathrm{~K}$, at which temperature the metastable SkL is short-lived ( $<10 \mathrm{~s}$; see Fig. $3 \mathrm{~b})$ and can therefore relax into the conical state within the pulse duration. The reversible electric switching of the topological/non-topological magnetic structure is thus feasible, as manifested in the switching of $\rho_{y x}$ (Fig. 4b,c). The switching exhibits good repeatability (Fig. 4d), highlighting the deterministic nature of the working principle; that is, whereas thermal agitation at high temperatures allows the system to relax into the most thermodynamically stable state within a relatively short time, such relaxation is almost unexpected at low temperatures because the topological stability surpasses the thermal agitation. Whereas such a deterministic switching using heat pulses 
and quenching has often been demonstrated in glass-forming systems ${ }^{23-25}$, the switching between distinct long-range magnetic orders has, to the best of our knowledge, never been reported.

Our observations clearly establish that a metastable SkL hidden behind non-topological magnetic ordering can be accessed by applying sufficiently high cooling rates. Because the local moments generally develop as the temperature decreases, SkLderived physical properties become more accessible in the quenched SkL, facilitating experimental studies (for instance, the topological Hall resistivity is markedly enhanced at low temperatures; see Supplementary Fig. 6 and ref. 17). In the context of the possible application of skyrmions, in which the manipulation of a single skyrmion is ultimately considered ${ }^{26-30}$, we conjecture that a similar creation/annihilation method is also applicable to the case of a small number of skyrmions because the working principle demonstrated above is predicated on manipulating the delicate balance among the topological stability, the thermodynamic stability, and the thermal agitation, properties that remain well defined in a finite-size system. We have also shown that the topological stability is undermined at high temperatures, suggesting that the operating temperature of skyrmion devices must be chosen in the context of a competition between the topological stability and the thermal agitation.

\section{Methods}

Methods and any associated references are available in the online version of the paper.

Received 1 June 2015; accepted 10 September 2015; published online 12 October 2015; corrected after print 14 April 2016

\section{References}

1. Mermin, N. D. The topological theory of defects in ordered media. Rev. Mod. Phys. 51, 591-648 (1979).

2. Michel, L. Symmetry defects and broken symmetry. Configurations hidden symmetry. Rev. Mod. Phys. 52, 617-651 (1980).

3. Bogdanov, A. \& Yablonskii, D. A. Thermodynamically stable "vortices" in magnetically ordered crystals. The mixed state of magnets. Sov. Phys. JETP 68, 101-103 (1989).

4. Bogdanov, A. \& Hubert, A. Thermodynamically stable magnetic vortex states in magnetic crystals. J. Magn. Magn. Mater. 138, 255-269 (1994).

5. Mühlbauer, S. et al. Skyrmion lattice in a chiral magnet. Science 323, 915-919 (2009).

6. Münzer, W. et al. Skyrmion lattice in the doped semiconductor $\mathrm{Fe}_{1-x} \mathrm{Co}_{x} \mathrm{Si}$. Phys. Rev. B 81, R041203 (2010).

7. Moskvin, E. et al. Complex chiral modulations in FeGe close to magnetic ordering. Phys. Rev. Lett. 110, 077207 (2013).

8. Yu, X. Z. et al. Real-space observation of a two-dimensional skyrmion crystal. Nature 465, 901-904 (2010).

9. Yu, X. Z. et al. Near room-temperature formation of a skyrmion crystal in thin-films of the helimagnet FeGe. Nature Mater. 10, 106-109 (2011).

10. Pfleiderer, C. et al. Skyrmion lattices in metallic and semiconducting B20 transition metal compounds. J. Phys. Condens. Matter 22, 164207 (2010).

11. Bauer, A. et al. Quantum phase transitions in single-crystal $\mathrm{Mn}_{1-x} \mathrm{Fe}_{x} \mathrm{Si}$ and $\mathrm{Mn}_{1-x} \mathrm{Co}_{x} \mathrm{Si}$ : Crystal growth, magnetization, ac susceptibility, and specific heat. Phys. Rev. B 82, 064404 (2010).
12. Seki, S., Yu, X. Z., Ishiwata, S. \& Tokura, Y. Observation of skyrmions in a multiferroic material. Science 336, 198-201 (2012).

13. Franz, C. et al. Real-space and reciprocal-space Berry phases in the Hall effect of $\mathrm{Mn}_{1-x} \mathrm{Fe}_{x} \mathrm{Si}$. Phys. Rev. Lett. 112, 186601 (2014).

14. Tokunaga, Y. et al. A new class of chiral materials hosting magnetic skyrmions beyond room temperature. Nature Commun. 6, 7638 (2015).

15. Bundy, F. P. et al. The pressure-temperature phase and transformation diagram for carbon; updated through 1994. Carbon 34, 141-153 (1996).

16. Blatov, V. A., Shevchenko, A. P. \& Proserpio, D. M. Applied topological analysis of crystal structures with the program package ToposPro. Cryst. Growth Des. 14, 3576-3586 (2014)

17. Ritz, R. et al. Giant generic topological Hall resistivity of $\mathrm{MnSi}$ under pressure Phys. Rev. B 87, 134424 (2013).

18. Lee, M., Kang, W., Onose, Y., Tokura, Y. \& Ong, N. P. Unusual Hall effect anomaly in MnSi under pressure. Phys. Rev. Lett. 102, 186601 (2009).

19. Neubauer, A. et al. Topological Hall effect in the A phase of MnSi. Phys. Rev. Lett. 102, 186602 (2009).

20. Ye, J. et al. Berry phase theory of the anomalous Hall effect: Application to colossal magnetoresistance manganites. Phys. Rev. Lett. 83, 3737-3740 (1999).

21. Bruno, P., Dugaev, V. K. \& Taillefumier, M. Topological Hall effect and Berry phase in magnetic nanostructures. Phys. Rev. Lett. 93, 096806 (2004).

22. Milde, P. et al. Unwinding of a Skyrmion lattice by magnetic monopoles. Science 340, 1076-1080 (2013).

23. Wuttig, M. \& Yamada, N. Phase-change materials for rewriteable data storage. Nature Mater. 6, 824-832 (2007).

24. Raoux, S. Phase change materials. Annu. Rev. Mater. Res. 39, 25-48 (2009).

25. Oike, H. et al. Phase-change memory function of correlated electrons in organic conductors. Phys. Rev. B 91, R041101 (2015).

26. Romming, N. et al. Writing and deleting single magnetic skyrmions. Science 341, 636-639 (2013)

27. Iwasaki, J., Mochizuki, M. \& Nagaosa, N. Current-induced skyrmion dynamics in constricted geometries. Nature Nanotech. 8, 742-747 (2013).

28. Sampaio, J., Cros, V., Rohart, S., Thiaville, A. \& Fert, A. Nucleation, stability and current-induced motion of isolated magnetic skyrmions in nanostructures. Nature Nanotech. 8, 839-844 (2013).

29. Koshibae, W. et al. Memory functions of magnetic skyrmions. Jpn. J. Appl. Phys. 54, 053001 (2015)

30. Romming, N., Kubetzka, A., Hanneken, C., Bergmann, K. V. \& Wiesendanger, R. Field-dependent size and shape of single magnetic skyrmions. Phys. Rev. Lett. 114, 177203 (2015).

\section{Acknowledgements}

We thank N. Nagaosa, W. Koshibae and A. Rosch for fruitful discussions. This work was partially supported by JSPS KAKENHI (Grant Nos. 25220709, 24224009, 15H05459).

\section{Author contributions}

H.O. conducted all experiments and analysed the data. A.K. grew the single crystals used for the study. F.K. planned and supervised the project. H.O. and F.K. wrote the letter. All authors discussed the results and commented on the manuscript.

\section{Additional information}

Supplementary information is available in the online version of the paper. Reprints and permissions information is available online at www.nature.com/reprints.

Correspondence and requests for materials should be addressed to F.K.

\section{Competing financial interests}

The authors declare no competing financial interests. 


\section{Methods}

Sample preparation. A single crystal of MnSi was grown by the Czochralski method. The sample was oriented by Laue X-ray diffraction, cut with a wire saw, and polished to a size of $2.5 \times 0.95 \times$ $0.1 \mathrm{~mm}^{3}$, with the largest surface normal to the $\langle 100\rangle$ axis. The residual resistivity ratio of the sample used in this study was approximately 55 . $\mathrm{Cu}$ current leads of $0.43 \mathrm{~mm}$ diameter were attached to the sample and fixed with silver paste. Au wires for the voltage probe were soldered to the sample with indium. At $10 \mathrm{~K}$, the sum of the contact resistances of the two current electrodes is approximately $615 \mathrm{~m} \Omega$, whereas the resistance of the whole sample is only approximately $1.6 \mathrm{~m} \Omega$. Hence, the Joule heating during the current-pulse application occurs mainly at the contacts. For details on the estimation of the Joule heating and the subsequent heating and cooling rates, see Supplementary Information.
Transport measurements. The Hall resistivity $\rho_{y x}$ was measured at a current excitation of $33 \mathrm{~Hz}$ with the five-probe method under a magnetic field parallel to the $\langle 100\rangle$ axis using a lock-in amplifier (Signal Recovery, 7270 DSP) equipped with a transformer preamplifier (Stanford Research Systems, SR554). The current density of the a.c. excitation was set to less than $1.4 \times 10^{5} \mathrm{~A} \mathrm{~m}^{-2}$. We confirmed that the a.c. current caused no appreciable increase in the sample temperature. The pulse currents used for the sample heating were generated by a function generator (NF Corporation, WF1947) connected to a bipolar amplifier (NF Corporation, HSA4014). The voltage drop between the voltage probes attached to the sample was amplified with a low-noise preamplifier (NF Corporation, LI-75A) and was monitored as a function of time using a data logger (Data Translation, DT8824), so that we could derive the time-varying sample temperature (see Supplementary Information). During the time-varying Hall-resistivity measurements, we monitored the digital-to-analog converter output of the lock-in amplifier using the data logger. 


\section{Corrigendum: Interplay between topological and thermodynamic stability in a}

metastable magnetic skyrmion lattice

Hiroshi Oike, Akiko Kikkawa, Naoya Kanazawa, Yasujiro Taguchi, Masashi Kawasaki, Yoshinori Tokura and Fumitaka Kagawa

Nature Physics 12, 62-66 (2016); published online 12 October 2015; corrected after print 14 April 2016.

In the version of this Letter originally published the pulse heights in the pulse sequence in Figure $4 \mathrm{c}$ were incorrect. This has now been corrected in the online versions of the Letter. 